University of South Carolina

Scholar Commons

$4-15-2007$

\title{
Optically Induced Suppression of Spin Relaxation in Two- Dimensional Electron Systems with Rashba Interaction
}

Yuriy V. Pershin Dr

University of South Carolina - Columbia, pershin@physics.sc.edu

Follow this and additional works at: https://scholarcommons.sc.edu/phys_facpub

Part of the Physics Commons

Publication Info

Published in Physical Review B, ed. Gene D. Sprouse, Volume 75, Issue 16, 2007, pages

165320-1-165320-5.

Pershin, Y. V. (2007). Optically induced suppression of spin relaxation in two-dimensional electron systems with Rashba interaction. Physical Review B, 75(16), 165320-1 - 165320-5. DOI: 10.1103/

PhysRevB.75.165320

(c) Physical Review B, 2007, American Physical Society

This Article is brought to you by the Physics and Astronomy, Department of at Scholar Commons. It has been accepted for inclusion in Faculty Publications by an authorized administrator of Scholar Commons. For more information, please contact digres@mailbox.sc.edu. 


\title{
Optically induced suppression of spin relaxation in two-dimensional electron systems with Rashba interaction
}

\author{
Yuriy V. Pershin* \\ Department of Physics and Astronomy, Michigan State University, East Lansing, Michigan 48824-2320, USA
}

(Received 27 February 2007; published 24 April 2007)

\begin{abstract}
A pulsed technique for electrons in two-dimensional systems, in some ways analogous to spin echo in nuclear magnetic resonance, is discussed. We show that a sequence of optical below-band-gap pulses can be used to suppress the electron spin relaxation due to the D'yakonov-Perel' spin relaxation mechanism. The spin relaxation time is calculated for several pulse sequences within a Monte Carlo simulation scheme. The maximum of the spin relaxation time as a function of magnitude or width of the pulses corresponds to a $\pi$ pulse. It is important that even relatively distant pulses efficiently suppress spin relaxation.
\end{abstract}

DOI: 10.1103/PhysRevB.75.165320

PACS number(s): 72.15.Lh, 76.60.Lz, 85.75.-d

\section{INTRODUCTION}

There has been a lot of experimental ${ }^{1-5}$ and theoretical ${ }^{6-15}$ interest in the physics of spin relaxation in semiconductor structures. The main reason for that is the potential for spintronic applications. ${ }^{16-23}$ Controlling the spin relaxation rate is interesting from both fundamental and practical points of view. One of the ways through which spin polarization can be lost is spin-orbit interaction. Of particular interest is the Rashba spin-orbit (SO) interaction, ${ }^{24}$ which is observed in asymmetric heterostructures. The corresponding spin relaxation mechanism is known as the D'yakonov-Perel' spin relaxation mechanism. ${ }^{25}$

Let us consider a system of two-dimensional (2D) electrons confined in a quantum well or heterostructure. The Rashba spin-orbit interaction can be regarded as an effective momentum-dependent magnetic field acting on the electron spins. In the presence of the effective magnetic field, the electron spins experience a torque and precess in the plane perpendicular to the magnetic field direction with an angular frequency $\vec{\Omega}(\vec{k})$. This precession leads to an average spin relaxation (dephasing). Momentum scatterings reorient the direction of the precession axis, making the orientation of the effective magnetic field random and trajectory dependent. Therefore, frequent scattering events suppress the precession and consequently the spin relaxation. This is the motionalnarrowing behavior, according to which the spin relaxation time $\tau_{s}^{-1} \propto \tau_{p},{ }^{25}$ where $\tau_{p}$ is the momentum scattering time.

Spin echo is a standard way to overcome dephasing in nuclear magnetic resonance experiments. ${ }^{26}$ Nuclear spin magnetization, after a free induction decay, can be restored, as a result of the effective reversal of the dephasing of the spins (refocusing) by the application of a refocusing rf pulse (applied in a time shorter than or of the order of the $T_{2}$ time). Unfortunately, this method cannot be directly applied to electron spin coherence in heterostructures. One of the obstacles is that the minimum achievable rf pulse length of $\sim 10 \mathrm{~ns}$ is of the order of or even longer than the typical spin coherence time. Moreover, the effective magnetic field due to SO interaction is fixed only between two consecutive scattering events. Therefore, a refocusing pulse sequence for electron spin coherence in heterostructures should have a pulse separation of the order of $\tau_{p}$ and pulse duration much shorter than $\tau_{p}$. In what follows we discuss a possible realization of such refocusing pulse sequence based on a method from femtosecond optics.

In this paper we consider the dynamics of electron spin polarization in a two-dimensional semiconductor structure like a quantum well or heterostructure under a train of intense optical below-band-gap circularly polarized pulses. Recent experiments have demonstrated that the effective magnetic field due to an optical below-band-gap pulse coherently rotates electron spins on a time scale of $\sim 150 \mathrm{fs},{ }^{27}$ which is much shorter than typical values of $\tau_{p}$ in clean structures. The mechanism of spin rotation is based on the optical Stark effect. ${ }^{28}$ Physically, the optical Stark effect in semiconductors is related to optically induced modification (dressing) of quantum states, ${ }^{28}$ including optically induced spin splitting. ${ }^{27,29}$ Since a below-band-gap laser does not excite real excitons, the optically induced spin splitting lasts only as long as the pump pulse. The purpose of the current investigation is to study the effect of the pulse sequence on the electron spin relaxation time in 2D quantum structures with dominant D'yakonov-Perel' spin relaxation mechanism. Electron spin rotations due to the pulse sequence result in partial compensation of the spin precession due to the Rashba interaction. Correspondingly, the electron spin relaxation time becomes longer.

The main idea of our approach is illustrated in Fig. 1. Let us consider the evolution of an electron spin (initially aligned with the $z$ axis) during a time interval between two consecutive scattering events. Using a semiclassical approach to

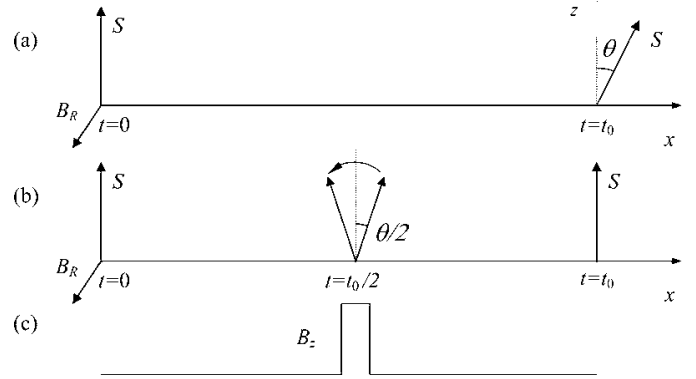

FIG. 1. Effect of an ideal $\pi$ pulse on the electron spin polarization vector $S$ : evolution of the spin polarization vector (a) without and (b) with a pulse; (c) pulse profile. 
electron space motion (the electrons are treated as classical particles in the effective-mass approximation), we assume that an electron moves along a straight trajectory with a constant velocity. Figure 1(a) shows that without a pulse, the direction of the electron spin at $t=t_{0}$ is changed by an angle $\theta$ due to precession around the effective spin-orbit magnetic field $B_{R}$. Figure $1(\mathrm{~b})$ demonstrates the effect of the light pulse applied at $t=t_{0} / 2$ [Fig. 1(c)] with such a width and intensity that the electron spin rotates around the $z$ axis by an angle $\pi$. It is readily seen that in this case at $t=t_{0}$ the electron spin is directed in the initial $z$ direction, so the effect of Rashba spin-orbit interaction is eliminated. In reality, of course, it is not possible to apply pulses exactly in the middle of each free flight interval for each electron; hence, a residual relaxation remains.

This paper is organized as follows. The Monte Carlo simulation scheme is introduced in Sec. II. In Sec. III we present the results of calculations. Section IV contains concluding remarks.

\section{CALCULATION SCHEME}

In order to get a quantitative estimation of the effect, we perform a Monte Carlo simulation of the spin dynamics in the presence of optical below-band-gap pulses. The electron spin relaxation time is calculated as a function of the electron spin precession angle $\varphi$ (due to a pulse) for different selected values of the spacing between pulses $T_{B}$ and for two types of pulse sequence: unidirectional and alternating. For the sake of simplicity, we assume that the effective magnetic field due to the pulse is much stronger than the effective magnetic field due to the spin-orbit interaction. This assumption allows us to consider the electron spin precession events due to the pulses as instantaneous.

Within a Monte Carlo simulation scheme, it is assumed that the electrons move along trajectories, which are defined by bulk scattering events (scattering on phonons, impurities, etc.), with an average velocity $v$. For the sake of simplicity, the scattering due to such events is assumed to be elastic and isotropic, i.e., the magnitude of the electron velocity is conserved in the scattering events, while the final direction of the velocity vector is randomly selected. The time scale of the bulk scattering events can then be fully characterized by a single rate parameter, the momentum relaxation time $\tau_{p}$, connected to the mean free path by $L_{p}=v \tau_{p}$.

The angular frequency corresponding to the Rashba coupling can be expressed as

$$
\vec{\Omega}=\eta \vec{v} \times \hat{z},
$$

where $\eta=2 \alpha m^{*} \hbar^{-2}, m^{*}$ is the effective electron mass, and $\alpha$ is the interaction constant that enters into the Rashba spinorbit coupling Hamiltonian

$$
H_{R}=\alpha \hbar^{-1}\left(\sigma_{x} p_{y}-\sigma_{y} p_{x}\right) .
$$

Here, $\vec{\sigma}$ is the Pauli matrix vector corresponding to the electron spin. The spin of a particle moving ballistically over a distance $1 / \eta$ will rotate by the angle $\gamma=1$. The angle of the spin rotation per mean free path $L_{p}$ is given by $\eta L_{p}$.
It is assumed that at the initial moment of time the electron spins are polarized in the $z$ direction (perpendicular to the plane) by a pump beam. We calculate $\langle\vec{S}\rangle$ as a function of time by averaging over an ensemble of electrons and taking into account both Rahsba-induced and optically induced spin precessions. The spin relaxation time is evaluated by fitting the time dependence of $\langle\vec{S}\rangle$ to an exponential decay. The detailed description of the basic Monte Carlo simulation scheme can be found in Ref. 6 .

We note that the selected Monte Carlo algorithm correctly describes the physics of the D'yakonov-Perel' spin relaxation mechanism. However, since all scattering parameters and temperature effects are taken into account via only two parameters $L_{p}$ and $\tau_{p}$, the temperature dependence as well as the role of Coulomb scattering cannot be easily evaluated, and more sophisticated simulations ${ }^{13}$ are required.

\section{RESULTS AND DISCUSSION}

The time dependence of $\langle\vec{S}\rangle$ was calculated for an ensemble of $10^{5}$ electrons for each value of the parameters describing the pulse sequence. The spin relaxation time for various pulse spacings is shown in Fig. 2 as a function of the spin rotation angle. We found that the rate of increase of spin relaxation time does not depend on the parameter $\eta L_{p}$ when $\eta L_{p}<1$. Instead, it is completely defined by the spacing between pulses, by the type of pulse sequence, and by the spin rotation angle due to a pulse.

A strong dependence of the spin relaxation time on the pulse sequence is observed. For short spacings between pulses, the unidirectional pulse sequence suppresses the spin relaxation more efficiently than the alternating pulse sequence. The spin relaxation time coincides for both pulse sequences only for $\varphi=\pi n$, where $n$ is an integer number. Furthermore, the spin relaxation time $\tau_{s}(\varphi)$ is a periodic function of $\varphi$ with period $2 \pi$, symmetric within a period $\tau_{s}(\pi+\beta)=\tau_{s}(\pi-\beta)$, where $\beta \in[0, \pi]$, and has a maximum at $\varphi=\pi(2 n+1)$. By increasing the spacing between pulses, the relaxation time decreases for both sequences. When the spacing between pulses becomes as long as a few momentum relaxation times, the spin rotations due to neighboring pulses become uncorrelated and the dependence of the spin relaxation time on $\varphi$ is the same for both pulse sequences. This is clearly seen for $T_{b}=3 \tau_{p}$ in Fig. 2 . It is important to notice that a significant increase of spin relaxation time is observed even when the spacing between pulses is longer than $\tau_{p}$, because it could be a minimal value of pulse period defined by laser specifications.

Figure 3 shows the spin relaxation time as a function of the spacing between pulses $T_{B}$ in the practically important situation $\varphi=\pi$, which is characterized by the longest spin relaxation time. The spin relaxation time sharply increases at small values of $T_{B}$ and slowly decreases with increase of $T_{B}$ to the spin relaxation time without pulses $\tau_{s}(\varphi=0)$.

Let us derive the asymptotic behavior of the spin relaxation time as a function of spacing between pulses in this case. First, consider the limit of distant pulses, when $T_{B}$ $\gg \tau_{p}$. Using a method described in Ref. 30 and assuming that 


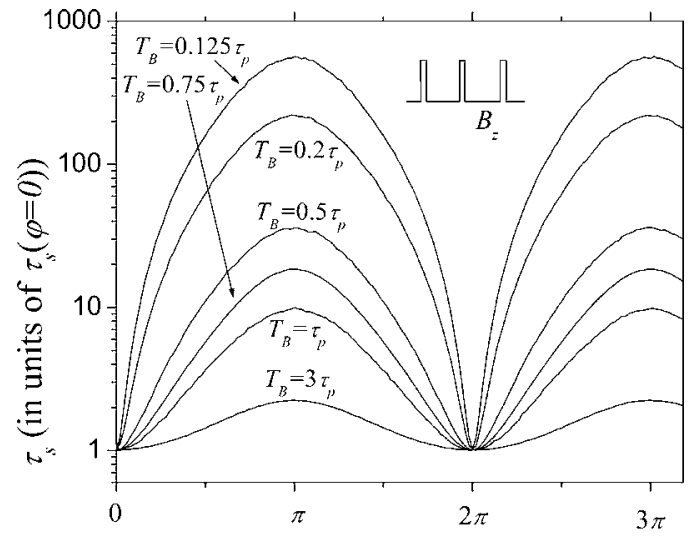

(a)

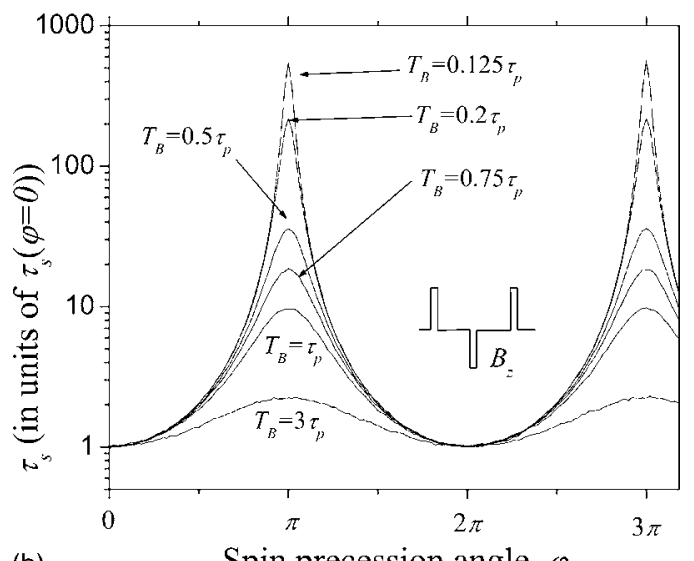

(b)

Spin precession angle, $\varphi$

FIG. 2. Spin relaxation time as a function of the spin precession angle $\varphi$ due to a pulse for several pulse periods $T_{B}$ and two types of pulse sequences: unidirectional (a) and, alternating (b) pulse sequence. These plots was obtained using the parameter value $\eta L_{p}$ $=0.4$.

a pulse is applied in an arbitrary time moment $t$ between two scattering events separated by a time interval $\tau$, the mean squared dephasing between these scattering events $\overline{\delta \theta^{2}}$ is given by

$$
\overline{\delta \theta^{2}}=\frac{1}{\tau} \int_{0}^{\tau} \Omega^{2}(2 t-\tau)^{2} d t=\frac{1}{3} \Omega^{2} \tau^{2} .
$$

The mean squared dephasing between two scattering events without a pulse is simply given by $\overline{\delta \theta^{2}}=\Omega^{2} \tau^{2}$. Taking into account the pulse probability, $\tau / T_{B}$, and the exponential distribution of probability of scattering,

$$
p(\tau, \tau+d \tau)=\left(1 / \tau_{p}\right) \exp \left(-\tau / \tau_{p}\right) d \tau,
$$

the mean free dephasing after $\overline{\delta \theta^{2}}$ scattering events will be

$$
\begin{aligned}
\overline{\delta \theta^{2}} & =n \frac{1}{\tau_{p}} \int_{0}^{\infty} e^{-\tau / \tau_{p}}\left[\left(1-\frac{\tau}{T_{B}}\right)+\frac{\tau}{T_{B}} \frac{1}{3}\right] \Omega^{2} \tau^{2} d \tau \\
& =2 n \Omega^{2} \tau_{p}^{2}\left(1-2 \frac{\tau_{p}}{T_{B}}\right) .
\end{aligned}
$$

If we take the relaxation time $\tau_{s}$ for a group of spins in phase

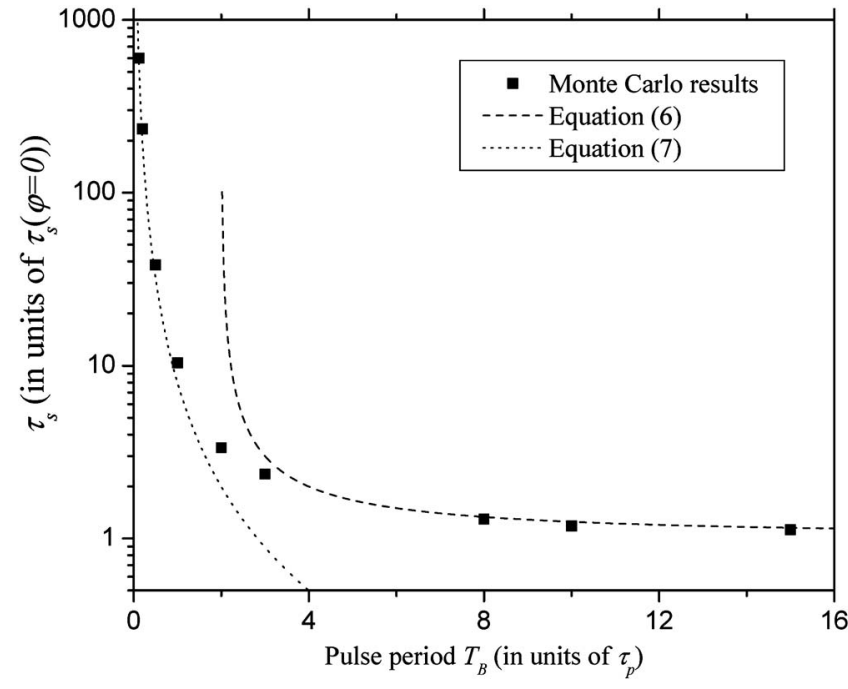

FIG. 3. Spin relaxation time as a function of the spacing between pulses $T_{B}$ at $\varphi=\pi$. The asymptotic behavior of the spin relaxation time [Eqs. (6) and (7)] is in excellent agreement with Monte Carlo results.

at the initial moment of time to get about $1 \mathrm{rad}$ out of step, we find

$$
\tau_{s}=\frac{\tau_{p}}{2 \Omega^{2} \tau_{p}^{2}} \frac{1}{1-2 \tau_{p} / T_{B}} .
$$

The first term at the right-hand side of Eq. (6) is the spin relaxation time without pulses; the second term describes the effect of the pulse sequence. In the opposite limit, when the number of pulses per mean free path is large, $T_{B} \ll \tau_{p}$, the spacing between pulses $T_{B}$ defines the characteristic angle of spin precession between two scattering events, instead of the momentum relaxation time $\tau_{p}$. Thus we can write

$$
\tau_{s} \sim \frac{\tau_{p}}{\Omega^{2} T_{B}^{2}} .
$$

The asymptotic expressions for the spin relaxation time, Eqs. (6) and (7), are presented in Fig. 3, showing an excellent agreement with Monte Carlo results.

We would like to emphasize that the proposed technique is most suitable for clean quantum structures with low electron density at low temperatures, i.e., when $\tau_{p}$ is long. For example, taking $v_{F}=5 \times 10^{6} \mathrm{~cm} / \mathrm{s}$ and $L_{p}=1 \mu \mathrm{m}$ we obtain $\tau_{p}=20 \mathrm{ps}$. Our calculations indicate that, in order to get a twofold increase in $\tau_{s}$, the spacing between the pulses at $\tau_{p}$ $=20 \mathrm{ps}$ should be $\sim 50 \mathrm{ps}$ at $\varphi=\pi$. The calculations presented in this paper have been made for a particular value of the parameter $\eta L_{p}=0.4$. This specific value of $\eta L_{p}$ is realizable in physical systems. For instance, considering an InAlAs/InGaAs quantum well ${ }^{31}$ with $\alpha=0.4 \times 10^{-12} \mathrm{eV} \mathrm{m}$, $m^{*}=0.04 m_{e}$, and $L_{p}=1 \mu \mathrm{m}$, we obtain $\eta L_{p}=0.42$. We would like to emphasize again that the rate of change of $\tau_{s}$ does not depend on $\eta L_{p}$ in the motional-narrowing regime.

From the experimental point of view, there are a couple of interrelated side effects that should be avoided. First of all, the experiment should be properly planned in order to mini- 


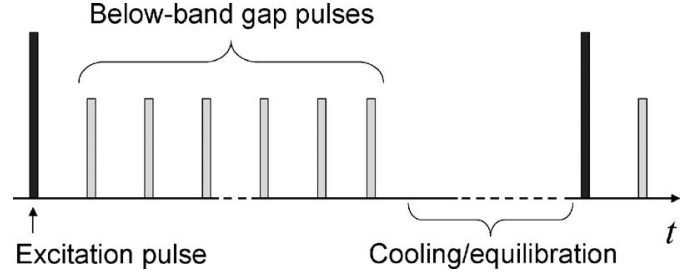

FIG. 4. Schematic representation of a possible pulse sequence reducing sample heating: the excitation pulse is followed by $M$ below-band-gap pulses and a time interval required for cooling.

mize the sample heating. This can be done in the following way (see Fig. 4 for details). The initial electron spin polarization can be excited $N$ times per second and followed by a group of $M$ below-band-gap pulses. All the remaining time between the last below-band-gap pulse in the group and the next pulse exciting spin polarization will be spent by the system in cooling and reaching a thermal equilibrium. The total number of pulses per second is $N M$. Typically, 1 $\lesssim \tau_{s}(\varphi=0) \lesssim 100 \mathrm{~ns}$. Therefore, the required number of pulses $M$ to monitor enhancement of $\tau_{s}(\varphi=0)$ at $T_{B}=50 \mathrm{ps}$ is $\sim \tau_{s}(\varphi=0) / T_{B}=20-20000$. In the recent experiment ${ }^{27}$ the sample was not significantly heated at $250 \mathrm{kHz}$ pump repetition rate. Consequently, the sample heating (per second) is smaller than in Ref. 27 if $N<[250000 / M]$, where [] denotes the integer part.

Another experimental effect, which might make it difficult to observe spin relaxation suppression, is related to undesirable excitation of real carriers. ${ }^{27}$ The energy of the below-band-gap laser must be adjusted to minimize the excitation of real carriers by compromising between statefilling effects and the magnitude of the Stark shift. ${ }^{27}$ Recent calculations for quantum dot geometry demonstrate that $\pi$ pulses may be obtained even for quite large detunings ( $\sim 70 \mathrm{meV}$ ) and experimentally realistic pump parameters. Definitely, the role of unwanted carrier excitation is smaller in systems with $\eta L_{p} \gtrsim 1$, when just a few pulses significantly increase $\tau_{s}$. It is expected that the real carrier excitation ef- fect should not be important when, at least, $M$ is equal to several tens.

\section{CONCLUSIONS}

In conclusion, the electron spin relaxation due to the D'yakonov-Perel' spin relaxation mechanism under a sequence of optical below-band-gap pulses was studied. The pulse sequence rotating electron spins around the axis perpendicular to the quantum well significantly suppresses spin relaxation in a way quite similar to the spin echo in nuclear magnetic resonance. The spin rotation mechanism is based on the optical Stark effect. It was demonstrated that the optical Start effect in semiconductors allows one to obtain very short ( $\sim 150 \mathrm{fs})$ and strong $(\sim 20 \mathrm{~T})$ pulses of effective magnetic field. ${ }^{27}$ The spin relaxation time was calculated for different pulse sequences and spacing between pulses. It was found that, in general, unidirectional pulse sequences suppress the spin relaxation more efficiently than the alternating one. Analytical formulas for the asymptotic behavior of the spin relaxation time were obtained. The proposed method of spin coherence control could find applications in probing spin coherence dynamics in heterostructures.

We would like to emphasize that the mechanism of suppression of spin relaxation described in this paper is not limited only to the Rashba-type spin-orbit interaction. Conceptually, the Dresselhaus-type spin-orbit interaction acts on electron spins in a similar way. Therefore, the mechanism of spin relaxation suppression by below-band-gap pulses could be used in systems with one of the above-mentioned types of spin-orbit interaction or with both of them.

\section{ACKNOWLEDGMENTS}

We gratefully acknowledge helpful discussions with C. Piermarocchi, V. Privman, and S. Saikin. This research was initiated at the Center for Quantum Device Technology at Clarkson University and was supported by the National Science Foundation, Grant No. DMR-0312491.
*Present address: Department of Physics, University of California, San Diego, La Jolla, California 92093-0319. Electronic address: pershin@physics.ucsd.edu

${ }^{1}$ J. M. Kikkawa and D. D. Awschalom, Nature (London) 397, 139 (1998); Phys. Rev. Lett. 80, 4313 (1998).

${ }^{2}$ H. Ohno, Science 281, 951 (1998).

${ }^{3}$ Y. Ohno, R. Terauchi, T. Adachi, F. Matsukura, and H. Ohno, Phys. Rev. Lett. 83, 4196 (1999).

${ }^{4}$ Z. Wilamowski, W. Jantsch, H. Malissa, and U. Rossler, Phys. Rev. B 66, 195315 (2002).

${ }^{5}$ A. M. Tyryshkin, S. A. Lyon, W. Jantsch, and F. Schaffler, Phys. Rev. Lett. 94, 126802 (2005).

${ }^{6}$ A. A. Kiselev and K. W. Kim, Phys. Rev. B 61, 13115 (2000).

${ }^{7}$ A. Bournel, P. Dollfus, E. Cassan, and P. Hesto, Appl. Phys. Lett. 77, 2346 (2000).

${ }^{8}$ S. Saikin, M. Shen, M.-C. Cheng, and V. Privman, J. Appl. Phys.
94, 1769 (2003).

${ }^{9}$ E. Ya. Sherman, Phys. Rev. B 67, 161303(R) (2003); Appl. Phys. Lett. 82, 209 (2003).

${ }^{10}$ O. Bleibaum, Phys. Rev. B 71, 195329 (2005).

${ }^{11}$ P. H. Song and K. W. Kim, Phys. Rev. B 66, 035207 (2002).

${ }^{12}$ F. X. Bronold, I. Martin, A. Saxena, and D. L. Smith, Phys. Rev. B 66, 233206 (2002).

${ }^{13}$ M. W. Wu and C. Z. Ning, Eur. Phys. J. B 18, 373 (2000); M. Q. Weng and M. W. Wu, Phys. Rev. B 68, 075312 (2003); M. Q. Weng, M. W. Wu, and L. Jiang, ibid. 69, 245320 (2004).

${ }^{14}$ M. M. Glazov and E. L. Ivchenko, JETP Lett. 75, 403 (2002).

${ }^{15}$ Yu. V. Pershin and V. Privman, Phys. Rev. Lett. 90, 256602 (2003); Phys. Rev. B 69, 073310 (2004); Yu. V. Pershin, ibid. 71, 155317 (2005); Yu. V. Pershin, Physica E (Amsterdam) 27, 77 (2005); 23, 226 (2004).

${ }^{16}$ S. Datta and B. Das, Appl. Phys. Lett. 56, 665 (1990). 
${ }^{17}$ M. Johnson, Science 260, 320 (1993).

${ }^{18}$ M. E. Flatté and G. Vignale, Appl. Phys. Lett. 78, 1273 (2001).

${ }^{19}$ I. Zutic, J. Fabian, and S. Das Sarma, Appl. Phys. Lett. 79, 1558 (2001).

${ }^{20}$ C. Ciuti, J. P. McGuire, and L. J. Sham, Appl. Phys. Lett. 81, 4781 (2002).

${ }^{21}$ T. Koga, J. Nitta, H. Takayanagi, and S. Datta, Phys. Rev. Lett. 88, 126601 (2002).

${ }^{22}$ X. F. Wang, P. Vasilopoulos, and F. M. Peeters, Phys. Rev. B 65, 165217 (2002)

${ }^{23}$ J. Fabian, I. Zutic, and S. Das Sarma, Phys. Rev. B 66, 165301 (2002).

${ }^{24}$ E. I. Rashba, Fiz. Tverd. Tela (Leningrad) 2, 1224 (1960) [Sov. Phys. Solid State 2, 1109 (1960)]; Y. A. Bychkov and E. I. Rashba, J. Phys. C 17, 6039 (1984).

${ }^{25}$ M. I. D'yakonov and V. I. Perel', Sov. Phys. Solid State 13, 3023
(1972); M. I. Dyakonov and V. Y. Kachorovskii, Sov. Phys. Semicond. 20, 110 (1986).

${ }^{26}$ A. Abragham, The Principles of Nuclear Magnetism (Oxford University Press, Oxford, 1961).

${ }^{27}$ J. A. Gupta, R. Knobel, N. Samarth, and D. D. Awschalom, Science 292, 2458 (2001).

${ }^{28}$ C. Cohen-Tannoudji and J. Dupont-Roc, Phys. Rev. A 5, 968 (1972); M. Combescot and R. Combescot, Phys. Rev. Lett. 61, 117 (1988).

${ }^{29}$ C. E. Pryor and M. E. Flatté, Appl. Phys. Lett. 88, 233108 (2006).

${ }^{30}$ C. P. Slichter, Principles of Magnetic Resonance, 3rd ed., Springer Series in Solid-State Sciences Vol. 1 (Springer-Verlag, Berlin, 1989), p. 212.

${ }^{31}$ T. Koga, J. Nitta, T. Akazaki, and H. Takayanagi, Phys. Rev. Lett. 89, 046801 (2002). 